Zhang, Z. and Chen, Q. 2009. "Prediction of particle deposition onto indoor surfaces by CFD with a modified Lagrangian method," Atmospheric Environment, 43(2), 319-328.

\title{
Prediction of Particle Deposition onto Indoor Surfaces by CFD with a Modified Lagrangian Method
}

\begin{abstract}
Z. ZHANG ${ }^{\mathrm{a}}$, Q. $\mathrm{CHEN}^{\mathrm{a} *}$
${ }^{a}$ National Air Transportation Center of Excellence for Research in the Intermodal Transport Environment (RITE), School of Mechanical Engineering, Purdue University, 585 Purdue Mall, West Lafayette, IN 47907-2088, USA
\end{abstract}

\begin{abstract}
Accurate prediction of particle deposition indoors is important to estimate exposure risk of building occupants to particulate matter. The prediction requires accurate modeling of airflow, turbulence, and interactions between particles and eddies close to indoor surfaces. This study used a $\overline{v^{\prime 2}}-f$ turbulence model with a modified Lagrangian method to predict the particle deposition in enclosed environments. The $\overline{v^{\prime 2}}-f$ model can accurately calculate the normal turbulence fluctuation $\overline{v^{\prime 2}}$, which mainly represents the anisotropy of turbulence near walls. Based on the predicted $\overline{v^{\prime 2}}$, we proposed an anisotropic particle-eddy interaction model for the prediction of particle deposition by the Lagrangian method. The model performance was assessed by comparing the computed particle deposition onto differently oriented surfaces with the experimental data in a turbulent channel flow and in a naturally convected cavity available from the literature. The predicted particle deposition velocities agreed reasonably with the experimental data for different sizes of particles ranging from $0.01 \mu \mathrm{m}$ to $50 \mu \mathrm{m}$ in diameter. This study concluded that the Lagrangian method can predict indoor particle deposition with reasonable accuracy provided the near-wall turbulence and its interactions with particles are correctly modeled.
\end{abstract}

Keywords: Particle deposition, Lagrangian method, CFD, v2f, indoor environment

\section{Introduction}

Scientific studies discovered significant association between the particle pollution and people's mortality and morbidity (Pope, 2000; Long et al., 2000). A recent research found that the chance of lung cancer death increased by $8 \%$ for every $10 \mu \mathrm{g} / \mathrm{m}^{3}$ increase of long-term fine particle exposure in the ambient air (Pope et al., 2002). Since people spend more than $90 \%$ of their time indoors and particle concentration indoors is often higher than that outdoors (He et al., 2005), exposure to indoor particulate matter (PM) can be a major threat to our health. Good understanding of the indoor particle exposure is thus necessary and important.

Particle deposition onto indoor surfaces can greatly alter the indoor particle exposure level. According to Nazaroff (2004), particle deposition removes $10 \mu \mathrm{m}$ particles 10 times and $2.5 \mu \mathrm{m}$

\footnotetext{
* Corresponding author. Tel.: +765 496 7562; E-mail address: yanchen@purdue.edu
} 
particles 1 time as much as the ventilation does, for typical indoor environments with one air exchange rate per hour. So the accurate modeling of particle deposition is crucial in predicting the actual PM exposure level in an indoor environment.

In general, there are two methods studying the particle deposition: experimental investigations and numerical simulations. Experimental investigations provide accurate indoor particle deposition data, such as those summarized by Lai (2002) and conducted more recently by Bouilly et al. (2005) and Lai and Nazaroff (2005). Although those measured deposition rates vary with particle sizes in a similar trend, they can differ by one to two orders of magnitude between different experiments. The large variations in measurements indicate that factors other than particle size also influence the particle deposition rates. Those factors can be the airflow pattern, turbulence level, and properties of indoor surfaces, etc. However, it is often very difficult to measure and report all those crucial information in experiment. So those measurements may not be comparable, and the interpretation of the large variations on deposition rates can be difficult.

Alternatively, the numerical modeling approach can accurately control most of those important factors, making the comparison and interpretation between numerical results viable and meaningful. However, to develop an accurate particle deposition model is very challenging, which requires accurate modeling of airflow, turbulence, and particle-eddy interactions. Commonly used airflow and particle models may not be adequate. For example, Tian and Ahmadi (2007) compared different model predictions of particle depositions in channel flows. The predicted particle deposition velocities, based on the popular k- $\varepsilon$ model, were higher than the measured data by one to four orders of magnitude for particles ranging 0.01 to $10 \mu \mathrm{m}$ in diameters. Such errors in deposition predictions can have a major impact on the prediction accuracy of PM exposure indoors. Therefore, to develop an accurate particle deposition model along with appropriate airflow model is necessary.

In this paper, we propose a numerical model for accurate prediction of particle deposition in indoor environments. The model is validated and evaluated by quality experimental data in the literature, and is used to analyze indoor particle deposition characteristics.

\section{Research methods}

\subsection{Brief review of modeling methods of predicting indoor particle deposition}

There are two modeling methods in predicting particle deposition: the Eulerian and Lagrangian methods. The Eulerian method treats particles as continuum and correlates particle deposition to airflow properties inside the concentration boundary layer. The airflow properties are input parameters of the deposition model and can be obtained analytically by assuming ideal airflow conditions and by fitting with experimental data. For example, Lai and Nazaroff (2000) developed an indoor particle deposition model that represents the state-of-the-art of such Eulerian method. By presuming an airflow parameter (i.e., the friction velocity) their model prediction agreed reasonably well with the experimental data by Cheng et al. (1997). However, the model prediction was less satisfactory in predicting particle deposition in another enclosed environment (Lai and Nazaroff, 2005). Such Eulerian deposition model is semi-empirical, and 
relies on the assumptions of the flow features inside the boundary layer. Due to the complexity of the indoor airflow, those assumptions could fail, and the performance and robustness of the Eulerian method can be affected.

Alternatively, the Lagrangian method tracks each particle directly based on the predicted airflow field by computational fluid dynamics (CFD), and avoids presuming flow conditions inside the boundary layer. However, the performance of the Lagrangian method is very sensitive to the accuracy of the predicted mean flow and turbulence, particularly near walls. The strict requirement on near-wall flow and turbulence modeling challenges the modeling capacity of many Reynolds-averaged-Navier-Stokes (RANS) models (Zhao et al., 2004; Zhang and Chen, 2006).

Effective near-wall treatment for the Lagrangian method is therefore necessary to the accurate prediction of particle deposition. Li and Ahmadi (1992) developed a near-wall model using DNS analysis of simple flows to quantify the wall-normal turbulent fluctuation within the viscous layer near a wall. Tian and Ahmadi (2007) successfully applied the near-wall model with a Reynolds stress model (RSM) to predict particle depositions in channel flows. Lai and Chen (2007) adopted a similar method with the RNG k- $\varepsilon$ turbulence model to predict indoor particle dispersion and deposition. The Lagrangian prediction of deposition fractions agreed with their Eulerian simulations. However, the deposition prediction was not further validated experimentally due to the lack of directly measured deposition data. Bouilly et al. (2005) conducted both Lagrangian simulations and experimental measurements of particle deposition rates in an indoor environment. They used large eddy simulation to predict the airflow and turbulence. The predicted deposition rates of coarse particles $(5 \mu \mathrm{m}$ and $10 \mu \mathrm{m}$ in diameters) agreed well with their measured data. Measured deposition rates for finer particles were reported, but no corresponding numerical results were provided. They indicated that further validation, especially for finer particles, was still necessary. More efforts need to be made in developing an accurate Lagrangian particle deposition model for both fine and coarse particles for indoor environments. This requires both suitable airflow and particle-eddy interaction models.

\subsection{Modeling of airflow and turbulence features by CFD}

Accurate prediction of airflow and turbulence is crucial to the success of modeling the particle deposition onto surfaces (Tian and Ahmadi, 2007). Zhai et al. (2007) and Zhang et al. (2007) evaluated a large variety of turbulence models in predicting airflow and turbulence in enclosed environments. These models covered a wide range of CFD approaches including RANS, detached eddy simulation, and large eddy simulation. Among these models, a modified $\overline{v^{\prime 2}}-f$ model (Lien and Kalitzin 2001; Davidson et al. 2003) had the best accuracy in predicting the mean flow and the turbulence. This study thus applied the $\overline{v^{\prime 2}}-f$ model (hereafter v2f-dav model) in predicting the airflows. The model formulation has the general form:

$$
\rho \frac{\partial \bar{\phi}}{\partial \mathrm{t}}+\rho \overline{\mathrm{u}_{\mathrm{j}}} \frac{\partial \bar{\phi}}{\partial \mathrm{x}_{\mathrm{j}}}-\frac{\partial}{\partial \mathrm{x}_{\mathrm{j}}}\left[\Gamma_{\phi, \mathrm{eff}} \frac{\partial \bar{\phi}}{\partial \mathrm{x}_{\mathrm{j}}}\right]=\mathrm{S}_{\phi},
$$

where $\phi$ represents independent flow variables, $\Gamma_{\phi, \text { eff }}$ the effective diffusion coefficient, $S_{\phi}$ the source term of an equation, and the over bars denote the Reynolds averaging. Table 1 
summarizes the mathematical form of each transport equation of the v2f-dav model. In Table 1, $\mathrm{u}_{\mathrm{i}}$ is the velocity component in i direction, $\mathrm{T}$ air temperature, $\mathrm{k}$ the kinetic energy of turbulence, $\varepsilon$ the dissipation rate of turbulent kinetic energy, $\overline{v^{\prime 2}}$ the wall-normal turbulence fluctuation, $\mathrm{p}$ air pressure, $\mathrm{H}$ air enthalpy, $\mu_{\mathrm{t}}$ eddy viscosity, $\mathrm{G}_{\phi}$ the turbulence production for $\phi, \mathrm{S}$ the rate of the strain, and $f$ is a part of the $\overline{v^{\prime 2}}$ source term that accounts for the non-local blocking effect near walls. Note that the $\mathrm{T}$ in $\overline{v^{\prime 2}}$ and $f$ equations represents the turbulence time scale.

The modeled $\overline{v^{\prime 2}}$ is different from the normal component of the Reynolds stress vector in $\mathrm{y}$ direction. It is only a scalar that behaves similar to the wall-normal Reynolds stress close to walls due to the mathematical treatment in the turbulence model (Durbin and Pettersson Reif, 2001). The forms of the transport equations and their associated boundary conditions assure $\overline{v^{\prime 2}}$ of correct near-wall features. The appropriate boundary conditions of the turbulence variables at wall are as follows:

$$
\mathrm{k}=\overline{\mathrm{v}^{\prime 2}}=\mathrm{f}=0, \varepsilon=2 \mathrm{v} \frac{\mathrm{k}}{\Delta \mathrm{y}^{2}},
$$

Where $\Delta \mathrm{y}$ is the wall distance.

\subsection{Modeling of particle motion by the Lagrangian method}

The Lagrangian method tracks particles by solving the following equations based on the predicted airflow field:

$$
\frac{\mathrm{du}_{\mathrm{p}, \mathrm{i}}}{\mathrm{dt}}=\frac{1}{\tau} \frac{\mathrm{C}_{\mathrm{D}} \mathrm{Re}_{\mathrm{p}}}{24}\left(\mathrm{u}_{\mathrm{i}}-\mathrm{u}_{\mathrm{p}, \mathrm{i}}\right)+\frac{\mathrm{g}_{\mathrm{i}}\left(\rho_{\mathrm{p}}-\rho\right)}{\rho_{\mathrm{p}}}+\mathrm{n}_{\mathrm{i}}(\mathrm{t})+\mathrm{F}_{\mathrm{L}, \mathrm{i}}+\mathrm{F}_{\mathrm{Th}, \mathrm{i}},
$$

The left hand side of the equation represents the inertial force per unit mass $\left(\mathrm{m} / \mathrm{s}^{2}\right)$, where $u_{\mathrm{p}, \mathrm{i}}$ is the particle velocity component in i direction. The first term on the right hand side is the drag term, where $\tau$ is the particle relaxation time, $C_{D}$ the drag coefficient, $\operatorname{Re}_{\mathrm{p}}$ the Reynolds number based on the particle diameter, particle velocity, and air velocity. The second term represents the gravity and buoyancy, where $\rho$ and $\rho_{p}$ are the density of the air and particles, respectively. The $\mathrm{n}_{\mathrm{i}}(\mathrm{t}), \mathrm{F}_{\mathrm{L}, \mathrm{i}}$ and $\mathrm{F}_{\mathrm{Th}, \mathrm{i}}$ are the Brownian force, the shear induced lift force, and the thermophoretic force on a unit mass basis, respectively.

The drag coefficient for a spherical particle was calculated according to Hinds (1982):

$$
C_{D}=\left\{\begin{array}{lc}
\frac{24}{\operatorname{Re}_{p}} & \operatorname{Re}_{p}<1 ; \\
\frac{24}{\operatorname{Re}_{p}}\left(1+0.15 \operatorname{Re}_{p}^{0.687}\right) & 1 \leq \operatorname{Re}_{p}<400 .
\end{array}\right.
$$

In general, the drag force is the dominant force on particles while the other forces can become important depending on the particle size and flow conditions. So these forces are included and modeled as follows: 


$$
\begin{gathered}
\mathrm{n}_{\mathrm{i}}(\mathrm{t})=\zeta \mathrm{i} \sqrt{\frac{216 \mu \sigma \mathrm{T}}{\pi \rho_{\mathrm{p}}^{2} \mathrm{C}_{\mathrm{c}} \Delta \mathrm{t}}} \cdot \mathrm{d}_{\mathrm{p}}^{-2.5}, \\
\mathrm{~F}_{\mathrm{L}, \mathrm{i}}=5.188 \frac{v^{1 / 2} \rho \mathrm{d}_{\mathrm{ij}}\left(\mathrm{u}_{\mathrm{i}}-\mathrm{u}_{\mathrm{p}, \mathrm{i}}\right)}{\rho_{\mathrm{p}}\left(\mathrm{d}_{\mathrm{lk}} \mathrm{d}_{\mathrm{kl}}\right)^{1 / 4}} \cdot \mathrm{d}_{\mathrm{p}}^{-1}, \\
\mathrm{~F}_{\mathrm{Th}, \mathrm{i}}=\frac{-36 \mu^{2} \mathrm{C}_{\mathrm{s}}\left(\mathrm{k} / \mathrm{k}_{\mathrm{p}}+\mathrm{C}_{\mathrm{t}} \mathrm{Kn}\right)}{\rho \rho_{\mathrm{p}}\left(1+3 \mathrm{C}_{\mathrm{m}} \mathrm{Kn}\right)\left(1+2 \mathrm{k} / \mathrm{k}_{\mathrm{p}}+2 \mathrm{C}_{\mathrm{t}} \mathrm{Kn}\right)} \frac{1}{\mathrm{~T}} \frac{\partial \mathrm{T}}{\partial \mathrm{x}_{\mathrm{i}}} \cdot \mathrm{d}_{\mathrm{p}}^{-2},
\end{gathered}
$$

where, $\zeta$ is a Gaussian random number with zero mean and unit variance, $\sigma$ is the StefanBoltzmann constant, $\Delta t$ is the time step for particle tracking, $d_{i j}$ is the rate of deformation tensor, $\mathrm{k}$ and $\mathrm{k}_{\mathrm{p}}$ are conduction coefficient of fluid and particle, and $\mathrm{Kn}$ is the Knudsen number. More detailed information of those forces is available in the FLUENT reference manual (FLUENT, 2005).

\subsection{Modeling the turbulent dispersion of particles and the particle-eddy interactions near walls}

Particle turbulent dispersion is associated with the instantaneous flow fluctuations and is an important mechanism of particle deposition. When particles are in the turbulent core and the turbulence is assumed to be isotropic, the discrete random walk model (DRW) can be used to model the particle turbulent dispersion. The isotropic DRW model correlates the instantaneous flow fluctuations with the turbulent kinetic energy $\mathrm{k}$ :

$$
\mathrm{u}_{\mathrm{i}}^{\prime}=\zeta_{\mathrm{i}} \sqrt{2 \mathrm{k} / 3}
$$

where $u_{i}^{\prime}$ is the modeled fluctuation velocity in i direction, $\zeta$ is a Gaussian random number with zero mean and unit variance. The sum of the $u_{i}^{\prime}$ and the mean velocity $\overline{u_{i}}$ was used as the instantaneous air velocity $u_{i}$ in equations (3) and (6).

Previous studies have confirmed that the isotropic DRW model is effective and accurate in modeling particle dispersion and distribution in various flows (Gosman and Ioannides, 1981; Lai and Chen, 2007; Zhang and Chen, 2007). However, directly applying the isotropic DRW model with a RANS model (e.g. standard k- $\varepsilon$ model) can lead to significant over prediction of particle deposition rate. This is because RANS models with simple wall treatment cannot predict the extremely anisotropic turbulence near wall. The turbulence fluctuation perpendicular to wall is damped out significantly faster than that in other directions close to a surface. Since the v2f-dav model directly predicted the wall-normal fluctuation $\overline{v^{\prime 2}}$ near wall, this investigation used the $\overline{v^{\prime 2}}$ profile to modify the isotropic DRW model. The modified DRW model accounted for the anisotropic particle-eddy interactions near wall, and can be expressed as:

$$
\begin{aligned}
& \mathrm{u}_{\perp}^{\prime}=\zeta_{\perp} \sqrt{\overline{v^{\prime 2}}} \\
& \mathrm{u}_{\|}^{\prime}=\zeta_{\|} \sqrt{\left(2 \mathrm{k}-\overline{v^{\prime 2}}\right) / 2} ; \quad \mathrm{y}^{+} \leq \mathrm{y}_{\mathrm{Lim}}^{+} \\
& \mathrm{u}_{\mathrm{i}}^{\prime}=\zeta_{\mathrm{i}} \sqrt{\overline{\mathrm{u}_{\mathrm{i}}^{\prime} \mathrm{u}_{\mathrm{i}}^{\prime}}}=\zeta_{\mathrm{i}} \sqrt{2 \mathrm{k} / 3} ; \quad \text { otherwise }
\end{aligned}
$$


where the subscripts ${ }_{\perp}$ and $\|$ denotes the spatial coordinates normal and parallel to a wall, and $\mathrm{y}_{\text {Lim }}^{+}$sets the upper bound of the anisotropic region.

The generated instantaneous fluctuations by equation (9) remained unchanged for a particle trajectory within a time that is the minimum of the eddy life time and the time needed for the particle to cross the eddy (FLUENT, 2005).

\subsection{Calculation of the particle deposition velocity from Lagrangian trajectories}

The particle deposition velocity measures the rate of the deposition process and is given as:

$$
\mathrm{V}_{\mathrm{d}}=\frac{\mathrm{J}}{\mathrm{C}_{\infty}}
$$

where $\mathrm{J}$ is the deposition flux onto a surface defined as the number of particles deposited per unit time and unit area of the surface, and $\mathrm{C}_{\infty}$ represents the mean particle concentration.

The study used two schemes to derive $\mathrm{V}_{\mathrm{d}}$ according to two different Lagrangian tracking strategies. For the first tracking strategy, particles are initially uniformly distributed in a domain and are released simultaneously. Each trajectory represents the path line of a single particle. When the trajectory hits the wall at time $t$, it indicates one particle deposits at that time as shown in Figure 1(a). The particle deposition velocity onto a surface can be estimated by counting the number of deposited trajectories in a time period:

$$
\mathrm{V}_{\mathrm{d}}=\frac{\mathrm{N}_{\mathrm{d}} /(\mathrm{t} \cdot \mathrm{A})}{\overline{\mathrm{N}} / \mathrm{V}}
$$

where $\mathrm{N}_{\mathrm{d}}$ is the number of trajectories deposited within time period $\mathrm{t}, \mathrm{A}$ is the area of the surface, $\overline{\mathrm{N}}$ is the time averaged number of particles in the space, and V is the volume of the space. Zhang and Ahmadi (2000) successfully applied such scheme in simulating particle deposition in channel flows.

For the second tracking strategy, particles are released simultaneously from a point source. Each trajectory represents a strike line and carries certain flow rate of particles. When the trajectory hits the wall at time $t$, succeeding particles will hit at the same location on the wall after time $t$ as illustrated in Figure 1(b). So the particle deposition flux J can be estimated as:

$$
J=\frac{\sum_{\mathrm{i}=1}^{\mathrm{n}} \dot{\mathrm{M}}_{\mathrm{i}}\left(\mathrm{t}_{\text {max }}-\mathrm{t}_{\mathrm{i}}^{\mathrm{dep}}\right)}{\mathrm{t}_{\text {max }} \mathrm{A}}
$$

where $n$ is the number of trajectories, $\dot{M}_{i}$ is the particle number flow rate carried by each trajectory $\mathrm{i}, \mathrm{t}_{\mathrm{i}}^{\mathrm{dep}}$ is the time at which trajectory $\mathrm{i}$ deposits, and $\mathrm{t}_{\max }$ is the maximum computing time for each particle. $t_{\max }$ was set to be identical for all trajectories. If a particle has not yet deposited at the end of the computation, $t_{i}^{d e p}=t_{\max }$. The averaged particle concentration at time $\mathrm{t}_{\max }$ can be estimated accordingly as: 


$$
\mathrm{C}_{\infty}=\frac{\sum_{\mathrm{i}=1}^{\mathrm{n}} \dot{\mathrm{M}}_{\mathrm{i}} \mathrm{t}_{\mathrm{i}}^{\mathrm{dep}}}{\mathrm{V}}
$$

Therefore, the particle deposition velocity can be also expressed as:

$$
\mathrm{V}_{\mathrm{d}}=\frac{\sum_{\mathrm{i}=1}^{\mathrm{n}}\left(\mathrm{t}_{\text {max }}-\mathrm{t}_{\mathrm{i}}^{\text {dep }}\right)}{\sum_{\mathrm{i}=1}^{\mathrm{n}} \mathrm{t}_{\mathrm{i}}^{\mathrm{dep}}} \frac{\mathrm{V}}{\mathrm{t}_{\text {max }} \mathrm{A}}
$$

The second tracking strategy is in accord with the experiments that release particles continuously during measurements (e.g., Thatcher et al., 1996) while the first strategy simulates the conditions that particles are premixed and particle concentration continues to decay during measurements (e.g., Bouilly et al., 2005). In the present study, we used both deposition calculation schemes to correspond to the tracking strategies and associated experimental conditions.

\subsection{Numerical procedures}

This study used commercial CFD software, FLUENT (version 6.2) to predict both air motion and particle deposition. The numerical procedure for the flow simulation was identical to that used by Zhang et al. (2007), and was not repeated here. For the particle simulations, the isotropic DRW model is not compatible with the anisotropic near-wall treatment as state in equation (9). The nearwall treatment was implemented into an anisotropic DRW model associated with a Reynolds stress model (RSM) by using user-defined functions. The anisotropic DRW model can be expressed as:

$$
u_{i}^{\prime}=\zeta_{i} \sqrt{\overline{u_{i}^{\prime} u_{i}^{\prime}}}
$$

where $\overline{u_{i}^{\prime} u_{i}^{\prime}}$ is the normal Reynolds stress component in i direction.

Hence, we used the v2f-dav model in predicting the airflow and adopted the RSM affiliated Lagrangian method to predict particle dispersion and deposition. Final status of each trajectory including its deposition time and position was recorded to calculate the particle deposition velocities using additional user-defined functions.

\section{Results and discussion}

This investigation first validated the deposition model in an isothermal turbulent channel flow because of quality experimental data available in the literature. The validated model was then applied to study particle deposition onto surfaces of different orientations in a naturally convected cavity. The experimental data by Thatcher et al. (1996) had superior quality and was used to further analyze and evaluate the model performance.

\subsection{Predicting particle deposition in a channel flow}

Figure 2(a) shows the geometry of the channel with $0.02 \mathrm{~m}$ in width and $0.4 \mathrm{~m}$ in length. The Reynolds number based on the mean flow and the channel width was 6667. Figures 2(b) and 2(c) show the computational grid distribution. The first grid centroid was $0.05 \mathrm{~mm}$ above the wall, 
which corresponded to about $y^{+}=1$. The computational domain and the flow conditions were in accord with those designed by Tian and Ahmadi (2007) in order to further compare with their simulations.

Figure 3 shows the comparison of predicted flow profiles at $\mathrm{X}=0.3 \mathrm{~m}$ downstream by the v2f-dav model and the DNS results by Kim et al. (1987). The predictions of the v2f-dav model agreed well with the DNS results in both the mean flow and its turbulence statistics. As a reference, this study also included the prediction of turbulence by the RNG k- $\varepsilon$ model and by the quadratic function used by Tian and Ahmadi (2007). The RNG k- $\varepsilon$ model predicted the correct k profile but not the $\overline{v^{\prime 2}}$ profile due to the isotropic assumption used. The quadratic function was only accurate in the viscous sublayer within $\mathrm{y}+<4$ above the wall.

For particle simulations, the study tracked particles of 26 different sizes between $0.01 \mu \mathrm{m}$ and $50 \mu \mathrm{m}$ in diameters. The density of all particles is $2450 \mathrm{~kg} / \mathrm{m}^{3}$. For each particle size, 30,000 particles were initially uniformly distributed within $\mathrm{y}+<30$ above the lower wall. Initial particle velocities were identical to the ambient airflow. Particle deposition velocities were calculated according to equation (11). An ensemble of 30,000 trajectories ensured sufficient statistical confidence of the predicted deposition velocities. Figure 4 shows the comparison of predicted results and experimental data from the literature collected by Sippola (2002). The deposition velocity and the particle relaxation time were normalized by the friction velocity $\mathrm{u}^{*}$ and the turbulence time scale $u^{* 2} / v$. Numerical predictions by Tian and Ahmadi (2007) were marked as grey lines, which were only approximate readings from their work for general comparison. One should refer to original paper for accurate data. Nevertheless, it is clear that particle deposition velocity can be dramatically over predicted based on RANS models without accurate prediction of the wall-normal fluctuation near walls. Because the v2f-dav model and the quadratic correction presented correct near-wall turbulent structures, both methods well predicted deposition velocities for particles of various sizes. The comparison confirmed that it is important to predict accurately the near-wall turbulence in order to correctly model the particle deposition by the Lagrangian method.

Comparison of Figures 4 (a) and (b) shows the impact of gravity on deposition for particles of different sizes. When particles are small with the dimensionless relaxation time less than $10^{-2}$, gravity does not have visible impact on the particle deposition. The downward gravitational settling becomes increasingly important for coarser particles, and the surface orientation greatly alters the deposition characteristics. Since an indoor environment consists of surfaces of different orientations, it is important that the particle model can accurately predict particle deposition onto differently oriented surfaces.

Although the deposition model with v2f-dav proposed here and that with the RSM model by Tian and Ahmadi (2007) are both accurate in turbulent channel flows, their computing costs are different. In fact, Zhang et al. (2007) reported that a RSM model requires about 2-4 times computing time compared with the v2f-dav model, while the RSM model could experience convergence difficulties in complicated indoor environments. Meanwhile, the v2f-dav model has better accuracy than the RSM model tested by Zhang et al. (2007) in different flow scenarios. Therefore, the particle deposition model based on the v2f-dav model is potentially suitable for indoor environmental studies and was further tested. 
3.2 Predicting particle deposition onto differently oriented surfaces in a naturally convected cavity

The particle model was validated in the previous section in an isothermal channel flow. Since the flow features in channel is simple and well understood, it is not surprising that the CFD simulations could reasonably predict the deposition behaviors. In contrast, the thermo-fluid conditions in indoor environments are more complicated and case dependent. The present case studied is to further test the model performance in predicting particle deposition onto differently oriented surfaces in a naturally convected indoor environment. Figure 5 presents the general information of the experimental chamber constructed by Thatcher et al. (1996). It is a cubic cavity with a vertical wall and the floor heated and with the ceiling and another wall cooled. The temperature differential between heated and cooled surfaces is $20 \mathrm{~K}$ while the front and back surfaces are well insulated and adiabatic. The Rayleigh number was about $4 \times 10^{9}$, similar to that of a typical full sized room with the wall-to-air temperature difference of $\pm 1.25 \mathrm{~K}$. The whole experimental chamber was carefully designed and controlled so that the surface temperature was uniform even at the corners. The thermal boundary conditions formed a counter-clockwise airflow in the cavity.

In the particle deposition experiment, highly mono-dispersed spherical particles were released from the cavity center. Overall particle concentration in the chamber was monitored and deposited particles onto four non-adiabatic surfaces were collected and analyzed to calculate the deposition velocity. Thatcher et al. (1996) measured particle deposition velocities in five different sizes including $0.1 \mu \mathrm{m}, 0.5 \mu \mathrm{m}, 0.7 \mu \mathrm{m}, 1.3 \mu \mathrm{m}$, and $2.5 \mu \mathrm{m}$ in diameters. All particles were solid ammonium fluorescein particles with a density of $1350 \mathrm{~kg} / \mathrm{m}^{3}$. Experiments were repeated for each particle size to ensure the statistical confidence of the experimental results.

The airflow simulation used boundary conditions in accord with the experiments. The computational domain was two-dimensional, containing $60 \times 60$ computing cells with refined mesh near all surfaces to capture the near-wall turbulence. The first grid point was $1 \mathrm{~mm}$ above the surfaces corresponding to $\mathrm{y}+=0.5$ to 1 . Predicted mean airflow pattern is shown in Figure 5, and the turbulence in Figure 6. The strongest turbulence occurred in the lower-left and upperright corners due to the confrontations of air streams with high temperature differential. The strong turbulence at corners partially explained that the measured particle deposition velocities were higher at these corners than at other regions in the experiment.

Further validation of the airflow and temperature distribution is difficult as the experimental study did not provide very detailed flow information. However, the performance of the v2f-dav model in the naturally convected space is reliable according to the recent study by Zhang et al. (2007). Then this investigation applied the Lagrangian method to predict the particle dispersion and deposition. The anisotropic near-wall treatment as stated in equation (9) were applied to $\mathrm{y}^{+}$ $<60$ region above the walls. The particle sizes studied were $0.1 \mu \mathrm{m}, 0.2 \mu \mathrm{m}, 0.3 \mu \mathrm{m}, 0.4 \mu \mathrm{m}$, $0.5 \mu \mathrm{m}, 0.7 \mu \mathrm{m}, 1.3 \mu \mathrm{m}, 2.2 \mu \mathrm{m}$, and $2.5 \mu \mathrm{m}$ in diameters, respectively, and 10,000 trajectories were tracked for each particle size. This study used a constant time step of $\Delta \mathrm{t}=10^{-3}$ (s) and tracked totally $2 \times 10^{5}$ time steps for each trajectory. The deposition velocities in the present case were calculated according to equation (14). 
Figure 7 compares the predicted and measured particle deposition velocities onto different surfaces. As a reference, the simulated results without considering Brownian force were also presented. In general, the predictions with and without the Brownian force are similar. Both the predictions agreed reasonably well with the measured data on all the surfaces while some differences still exist.

As shown in Figure 7(c), the predicted deposition rate without considering the Brownian force reduced to zero for particles smaller than $0.4 \mu \mathrm{m}$, which deviated from the measured data. In contrast, the Brownian force did not significantly alter the deposition rates on other surfaces for particles coarser than $0.1 \mu \mathrm{m}$ as shown in Figures 7 (a), (b) and (d). According to Li and Ahmadi (1992), the Brownian force was only significant in the deposition process for particles smaller than $0.05 \mu \mathrm{m}$ in diameter. The Brownian force above the floor is not expected to be comparable with the drag force for particles larger than $0.1 \mu \mathrm{m}$ in diameter. Therefore, the significance of the Brownian force in particle deposition onto the floor is likely associated with some unique flow features that only occurred near the floor.

Figure 8(a) illustrates the difference of the flow structures near the heated floor. The mean wallnormal velocity above the floor was pointing upwards within at least $10 \mathrm{~mm}$ above the floor. This was due to the upward buoyancy effect near the heated floor. The upward velocity led to upward drag to particles near the floor. For small particles whose gravity is negligible, the Brownian force became the only important force that can create downward motion and can deposit on the floor. Without considering the Brownian force, small particles are unlikely to deposit onto the floor. In contrast, the wall-normal velocity near the heated vertical wall was pointing towards the surface as shown in Figure 8(b). A particle could thus deposit to the wall due to the dominant wall-pointing drag force. The same theory could be applied to the cooled ceiling and wall. The near-wall drag force, rather than the Brownian force, determined the deposition rates for small particles on these surfaces.

The study also studied the importance of the thermophoretic force and the lift force in the deposition process. For the particles of 0.1-2.5 $\mu \mathrm{m}$ in diameters, the predicted deposition velocities onto all surfaces did not alter significantly by excluding either the thermophoretic or the lift force.

Meanwhile, the present numerical model predicted significant deposition on the warm wall for super micron particles. This finding agreed with the experimental data but not with the semiempirical Eulerian model by Nazaroff and Cass (1989), which would predict a low deposition rate. Thatcher et al. (1996) reported that the high flow instability led to high particle deposition rates in their measurements. In addition, our analysis showed that the wall-pointing airflow near the warm wall was also an important factor to the high particle deposition velocities. With such a complex flow in indoor environment, the accuracy of the semi-empirical Eulerian model can be affected. The Lagrangian method can have a better performance in predicting particle deposition.

Although the predicted particle depositions agreed in general with the measured data, the numerical results remarkably over-predicted the particle deposition velocities onto the warm wall for particles smaller than $0.7 \mu \mathrm{m}$. The error is likely attributed to the profile of wall-normal 
velocity near the warm wall and the experimental uncertainty. For example, the wall may not be perfectly vertical and temperature on wall may not be uniform, as suggested by Thatcher et al. (1996). However, without detailed experimental information, further evaluation and exact explanation are difficult.

\section{Conclusions}

This investigation proposed a new method for accurate prediction of particle deposition onto indoor surfaces. We used the $\overline{v^{\prime 2}}-f$ turbulence model to predict the airflow and turbulence, and proposed an anisotropic particle-eddy interaction model for the Lagrangian method to predict the particle deposition process.

The turbulence model and the modified Lagrangian method were tested and evaluated in the present study. The study found that accurate prediction of the airflow and turbulence, particularly close to walls, is very important to determine particle deposition onto surfaces by CFD with the Lagrangian method. The $\overline{v^{\prime 2}}-f$ turbulence model can accurately predict the airflow and nearwall turbulence. Based on the $\overline{v^{\prime 2}}-f$ model, the Lagrangian method with the anisotropic particle-eddy interaction model can predict the particle deposition velocities onto different surfaces with reasonable accuracy. This integrated airflow and particle model can be used to predict and analyze the particle deposition process in indoor environments.

Our analysis demonstrated the importance of surface properties, such as the surface orientation and the surface-to-air temperature differential, to the particle deposition. The surface orientation directly determined the impact of gravitational force on the particle deposition rates for coarse particles. The combination of surface orientation and surface-to-air temperature differential influenced the near-wall flow and turbulence structures, and can greatly affect particle-eddy interaction for fine particles near the surface. In the present study, the heated floor in the naturally convected space created a unique flow structure that made the Brownian effect dominant in determining particle deposition for particles up to $0.5 \mu \mathrm{m}$ in diameter. A particle deposition model thus needs to correctly resolve the detailed flow conditions near surfaces with different thermal and geometrical properties.

\section{Acknowledgement}

This project is funded by the U.S. Federal Aviation Administration (FAA) Office of Aerospace Medicine through the National Air Transportation Center of Excellence for Research in the Intermodal Transport Environment (RITE) Cooperative Agreement 04-C-ACE-PU-002. Although the FAA has sponsored this project, it neither endorses nor rejects the findings of this research. The presentation of this information is in the interest of invoking technical community comment on the results and conclusions of the research.

We would like to express our gratitude to Dr. Mark Sippola of the Lawrence Berkeley National Laboratories for kindly providing particle deposition data from his dissertation.

\section{References}


Bouilly, J., Limam, K., Beghein, D., Allard, F., 2005. Effect of ventilation strategies on particle decay rates indoors: an experimental and modelling study. Atmospheric Environment 39, 48854892 .

Cheng, Y.S., 1997. Wall deposition of radon progeny and particles in a spherical chamber. Aerosol Science and Technology 27, 131-146.

Davidson, L., Nielsen, P.V., Sveningsson, A., 2003. Modification of the V2F model for computing the flow in a 3D wall jet. Turbulence Heat and Mass Transfer 4, 577-584.

Durbin, P.A., Pettersson Reif, B.A., 2001. Statistical Theory and Modelling for Turbulent flow, John Wiley \& Sons Ltd., 171-181.

El-Shobokshy, M.S., 1983. Experimental measurements of aerosol deposition to smooth and rough surfaces. Atmospheric Environment 17, 639-644.

Friedlander, S.K., Johnstone, H.F., 1957. Deposition of suspended particles from turbulent gas streams. Industrial and Engineering Chemistry 49, 1151-1156.

Gosman, A.D., Ioannides, E., 1981. Aspects of computer simulation of liquid-fueled combustors. AIAA 19th Aerospace Sciences Meeting, AIAA-81-0323, St. Louis, Missouri.

He, C., Morawska, L., Gilbert, D., 2005. Particle deposition rates in residential houses. Atmospheric Environment 39(21), 3891-3899.

Hinds,W.C.,1984. Aerosol Technology: Properties, Behavior, and Measurement of Airborne Particles. NewYork:Wiley.

Kim, J., Moin, P., Moser, R.D., 1987. Turbulent statistics in fully developed channel flow at low Reynolds number. Journal of Fluid Mechanics 177, 133-166.

Kvasnak, W., Ahmadi, G., Bayer, R., Gaynes, M., 1993. Experimental investigation of dust particle deposition in a turbulent channel flow. Journal of Aerosol Science 24, 795-815.

Lai, C.K., 1997. An experimental study of the deposition of aerosol on rough surfaces and the implications for indoor air quality control. Ph.D. Dissertation, Imperial College, London, England.

Lai, A.C.K., Nazaroff, W.W., 2000. Modeling indoor particle deposition from turbulent flow onto smooth surfaces. Journal of Aerosol Science 31(4), 463-476.

Lai, A.C.K., 2002. Particle deposition indoors: a review. Indoor Air 12, 211-214.

Lai, A.C.K., Chen, F.Z., 2007. Comparison of a new Eulerian model with a modified Lagrangian approach for particle distribution and deposition indoors. Atmospheric Environment 41, 52495256. 
Lai, A.C.K., Nazaroff, W.W., 2005. Supermicron particle deposition from turbulent flow onto vertical surfaces with varying roughness. Atmospheric Environment 39, 4893 - 4900.

Lee, K.W., Gieseke, J.A., 1994. Deposition of particles in turbulent pipe flows. Journal of Aerosol Science 25, 699-709.

Li, A., Ahmadi, G., 1992. Dispersion and deposition of spherical particles from point sources in a turbulent channel flow. Aerosol Science and Technology 16, 209-226.

Lien, F., Kalitzin, G., 2001. Computations of transonic flow with the $\mathrm{v}^{2}$-f turbulence model. International Journal of Heat and Fluid Flow 22, 53-61.

Liu, B.Y.H., Agarwal, J.K., 1974. Experimental observation of aerosol deposition in turbulent flow. Aerosol Science 5, 145-155.

Long, C.M., Suh, H.H., Koutrakis, P., 2000. Characterization of indoor particle sources using continuous mass and size monitors. Journal of the Air and Waste Management Association 50, 1236-1250.

Montgomery, T.L., Corn, M., 1970. Aerosol deposition in a pipe with turbulent airflow. Aerosol Science 1, 185-213.

Nazaroff, W.W., Cass, G.R., 1989. Mass-transport aspects of pollutant removal at indoor surfaces. Environment International 15, 567-584.

Nazaroff, W.W., 2004. Indoor particle dynamics. Indoor Air 14, 175-183.

Pope, C.A., 2000. Review: Epidemiological basis for particulate air pollution health standards. Aerosol Science and Technology 32, 4-14.

Pope, C.A., Burnett, R.T., Thun, M.J., Calle, E.E., Krewski, D., Ito, K., Thurston, G.D., 2002. Lung cancer, cardiopulmonary mortality, and long-term exposure to fine particulate air pollution. Journal of the American Medical Association 287, 1132-1141.

Postma, A.K., Schwendiman, L.C., 1960. Studies in micrometrics: I. Particle deposition in conduits as a source of error in aerosol sampling. Report HW-65308, Richland, Washington: Hanford Laboratory.

Sehmel, G.A., 1968. Aerosol deposition from turbulent airstreams in vertical conduits. Report BNWL-578, Richland, Washington: Pacific Northwest Laboratory.

Sehmel, G.A., 1973. Particle eddy diffusivities and deposition velocities for isothermal flow and smooth surfaces. Aerosol Science 4, 125-138. 
Shimada, M., Okuyama, K., Asai, M., 1993. Deposition of submicron aerosol particles in turbulent and transitional flow. AIChE Journal 39, 17-26.

Sippola, M.R., 2002. Particle Deposition in Ventilation Ducts, Ph.D. dissertation, University of California, Berkeley, CA, USA.

Thatcher, T.L., Fairchild, W.A., Nazaroff, W.W., 1996. Particle deposition from natural convection enclosure flow onto smooth surfaces. Aerosol Science and Technology 25, 359-374.

Tian, L., Ahmadi, G., 2007. Particle deposition in turbulent duct flows - comparisons of different model predictions. Journal of Aerosol Science 38, 377-397.

Wells, A.C., Chamberlain, A.C., 1967. Transport of small particles to vertical surfaces. British Journal of Applied Physics 18, 1793-1799.

Zhai, Z., Zhang, Z., Zhang, W., Chen, Q., 2007. Evaluation of various turbulence models in predicting airflow and turbulence in enclosed environments by CFD: part-1: summary of prevalent turbulence models. HVAC\&R Research 13(6).

Zhang, H., Ahmadi, G., 2000. Aerosol particle transport and deposition in vertical and horizontal turbulent duct flows. Journal of Fluid Mechanics 406, 55-80.

Zhang, Z., Chen, Q., 2006. Experimental measurements and numerical simulations of particle transport and distribution in ventilated rooms. Atmospheric Environment 40(18), 3396-3408.

Zhang, Z., Chen, Q., 2007. Comparison of the Eulerian and Lagrangian methods for predicting particle transport in enclosed spaces. Atmospheric Environment 41(25), 5236-5248.

Zhang, Z., Zhang, W., Zhai, Z., Chen, Q., 2007. Evaluation of various turbulence models in predicting airflow and turbulence in enclosed environments by CFD: part-2: comparison with experimental data from literature. HVAC\&R Research 13(6).

Zhao, B., Zhang, Y., Li, X., Yang, X., Huang, D., 2004. Comparison of indoor aerosol particle concentration and deposition in different ventilated rooms by numerical method. Building and Environment 39(1), 1-8. 
(a) $\mathrm{h}=0.02 \mathrm{~m}$

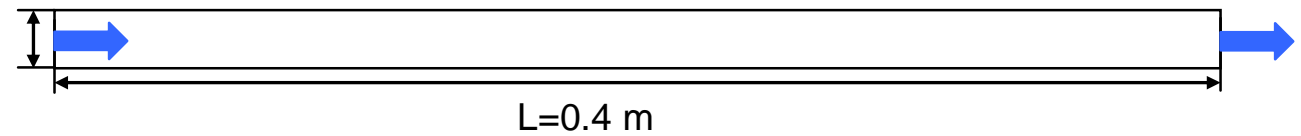

(b)

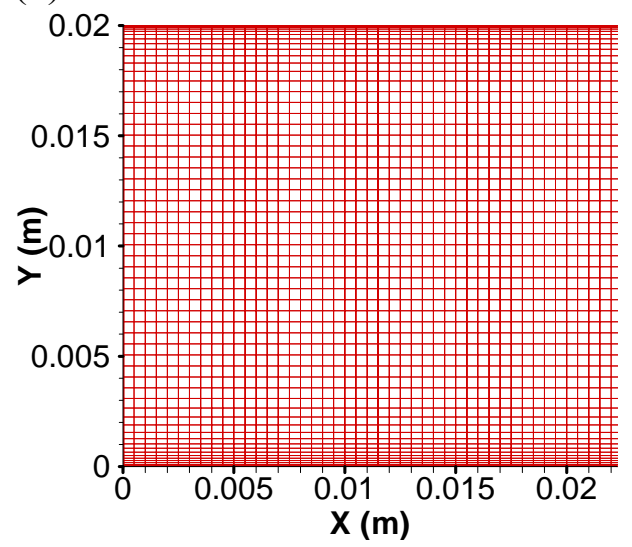

(c)

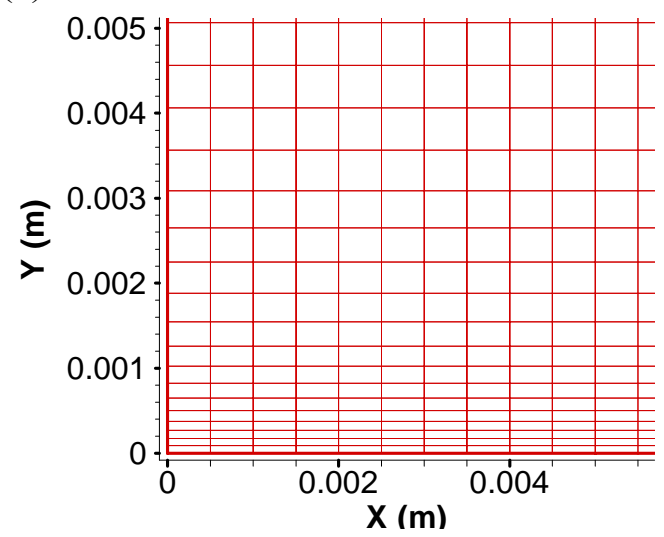

Figure 2. Geometrical and CFD grid information of the channel: (a) schematic view of the channel, (b) computing grid, (c) a closer view of the grid near the lower wall. 
(a)

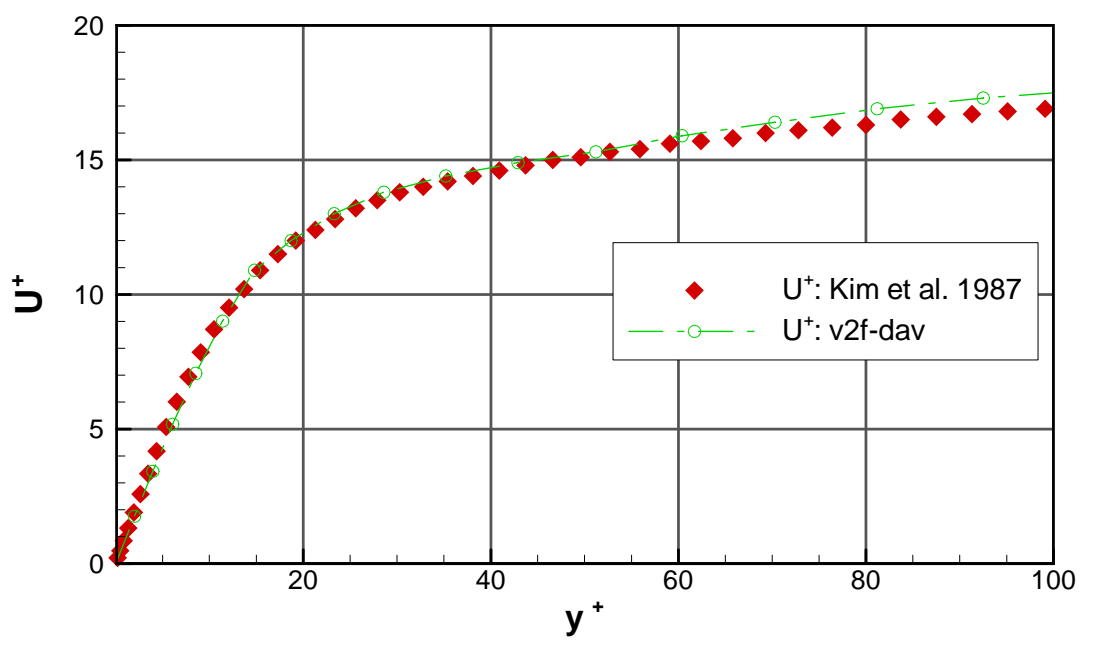

(b)

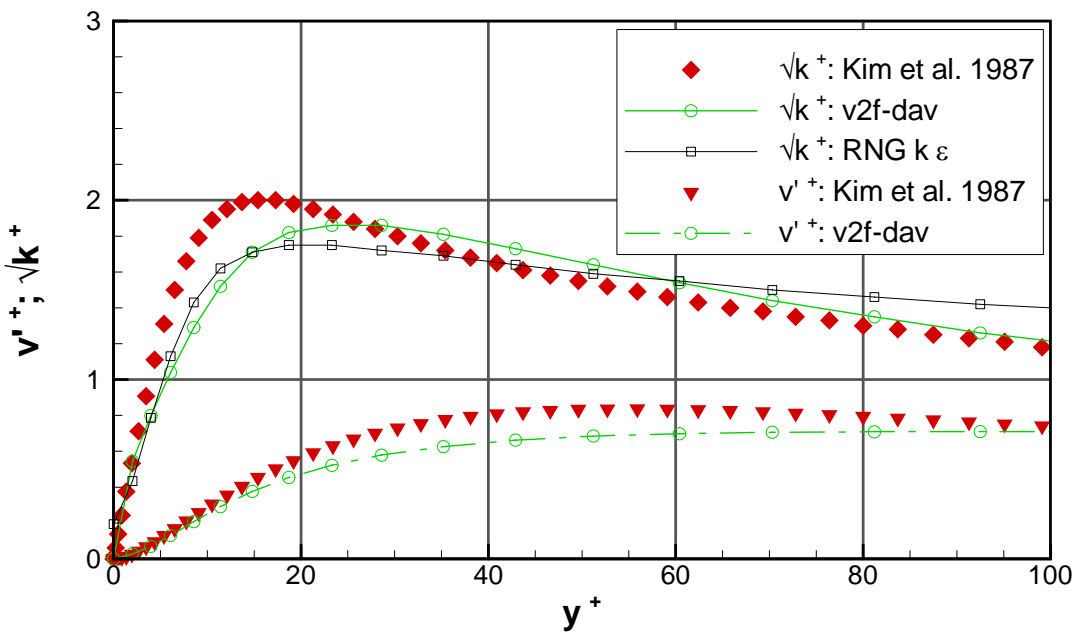

(c)

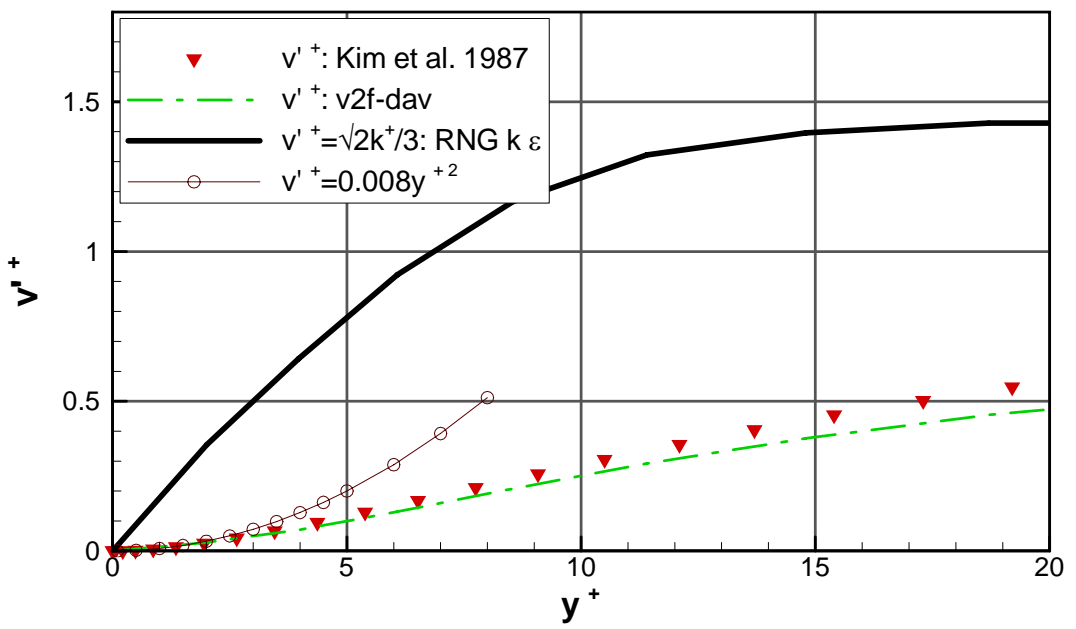

Figure 3. Comparison of predicted flow features with DNS results (Kim et al. 1987): (a) mean flow velocity, (b) turbulence, (c) a closer view of wall-normal turbulence fluctuation near wall. 
(a)

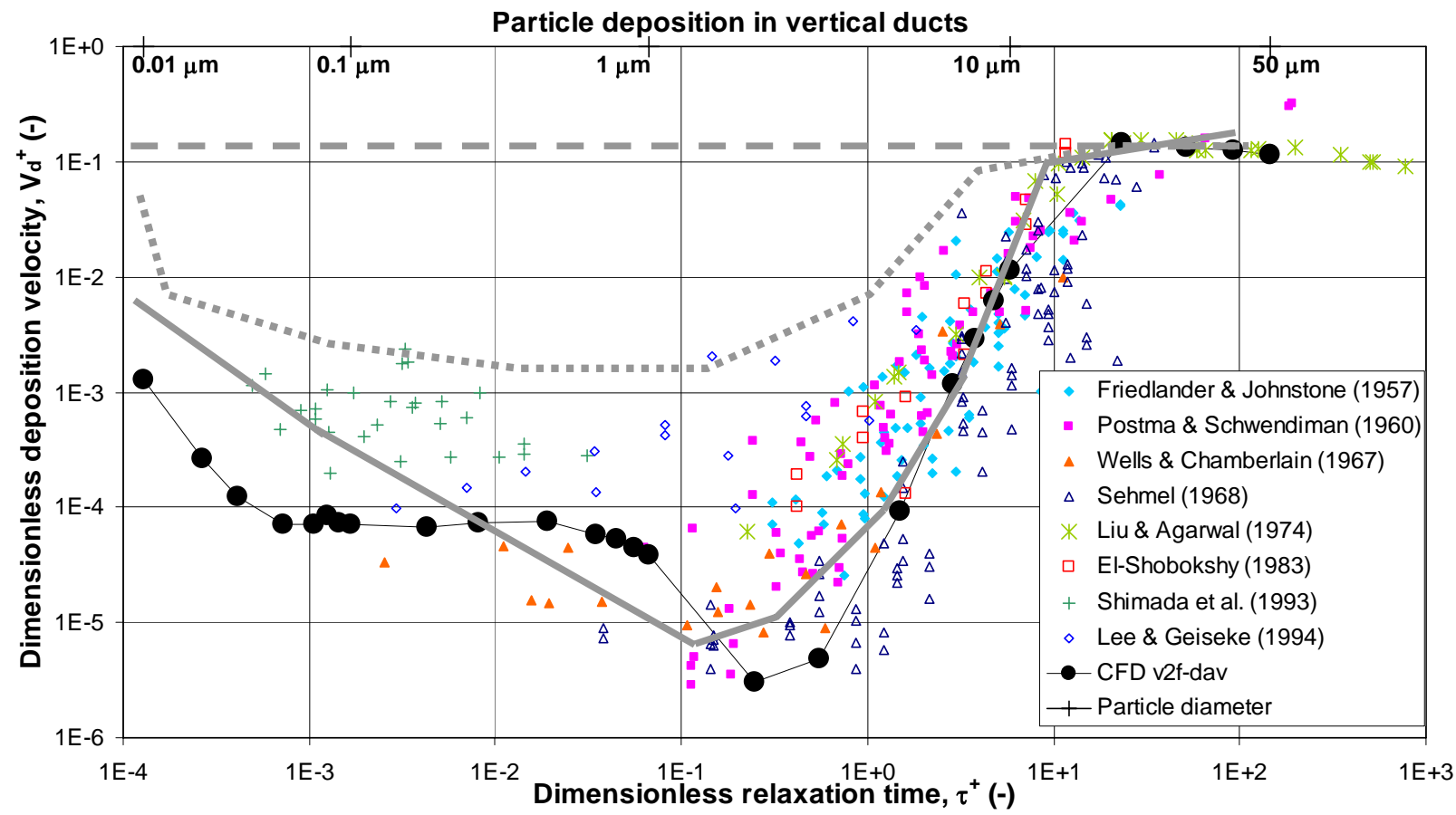

(b)

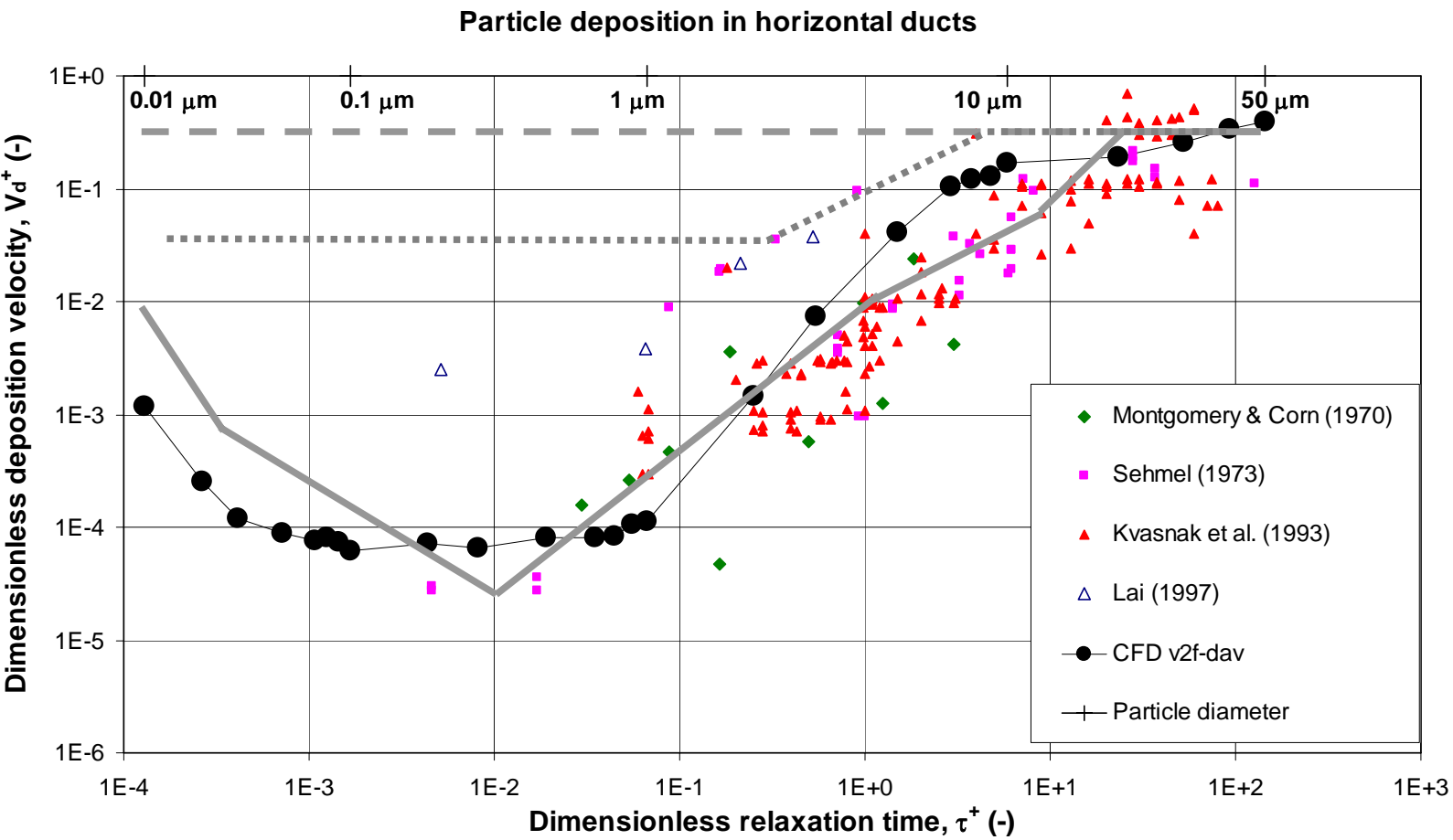

Figure 4. Comparison of predicted and measured particle deposition in the channel flow: (a) gravity in the flow direction, (b) gravity perpendicular to the flow direction. Measurement data readings were from Sippola (2002). The dashed, dotted, and solid grey lines were respectively the predictions of a k- $\varepsilon$ model with standard wall function and with a two layer wall function and a RSM model with the quadratic correction by Tian and Ahmadi (2007). 


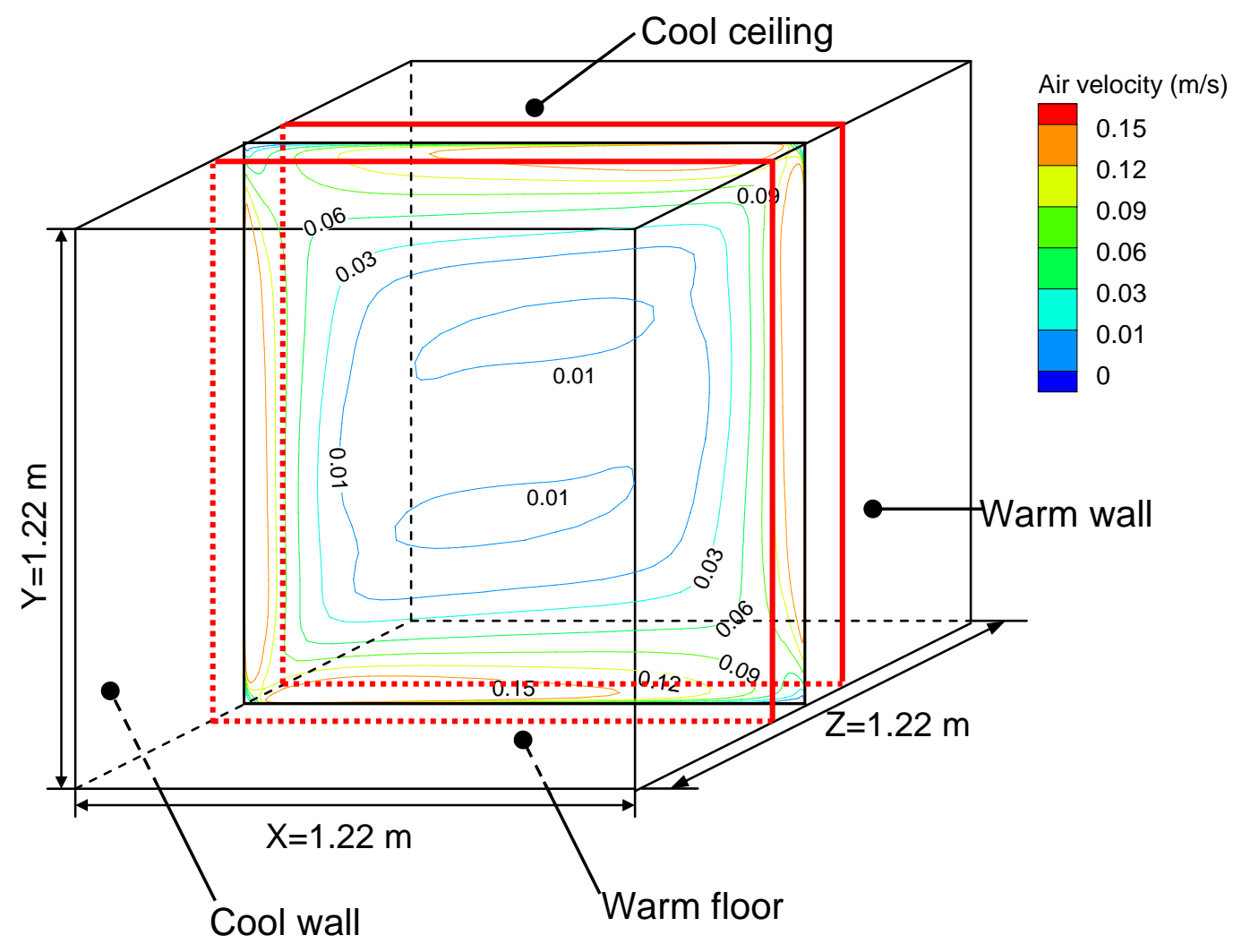

Figure 5. The configuration and airflow pattern of the naturally convected cavity (Thatcher et al. 1996). The two red frames enclosed the surface areas where deposited particles were collected in the experiment; the colored contours represent the magnitude of simulated air velocity $(\mathrm{m} / \mathrm{s})$ inside the cavity. 
(a)

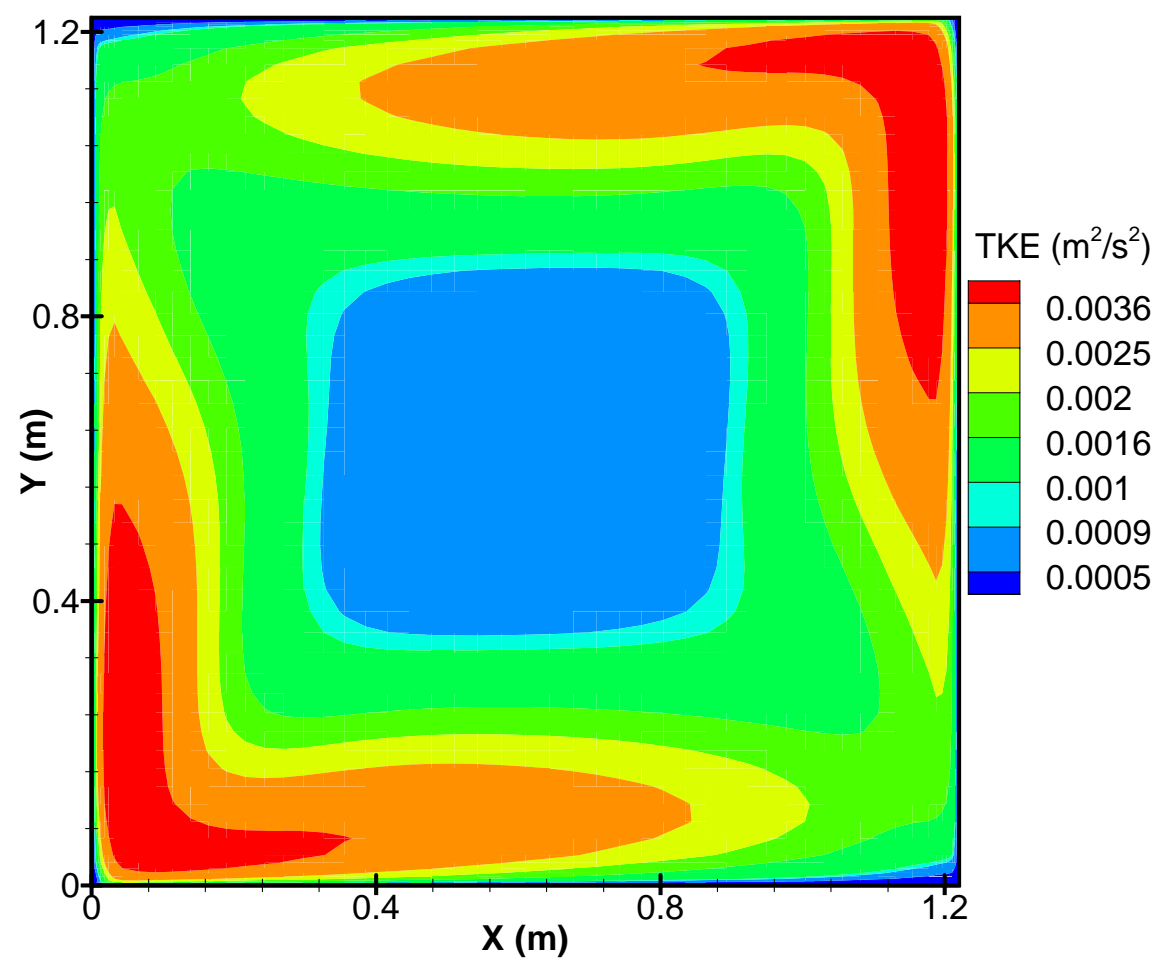

(b)

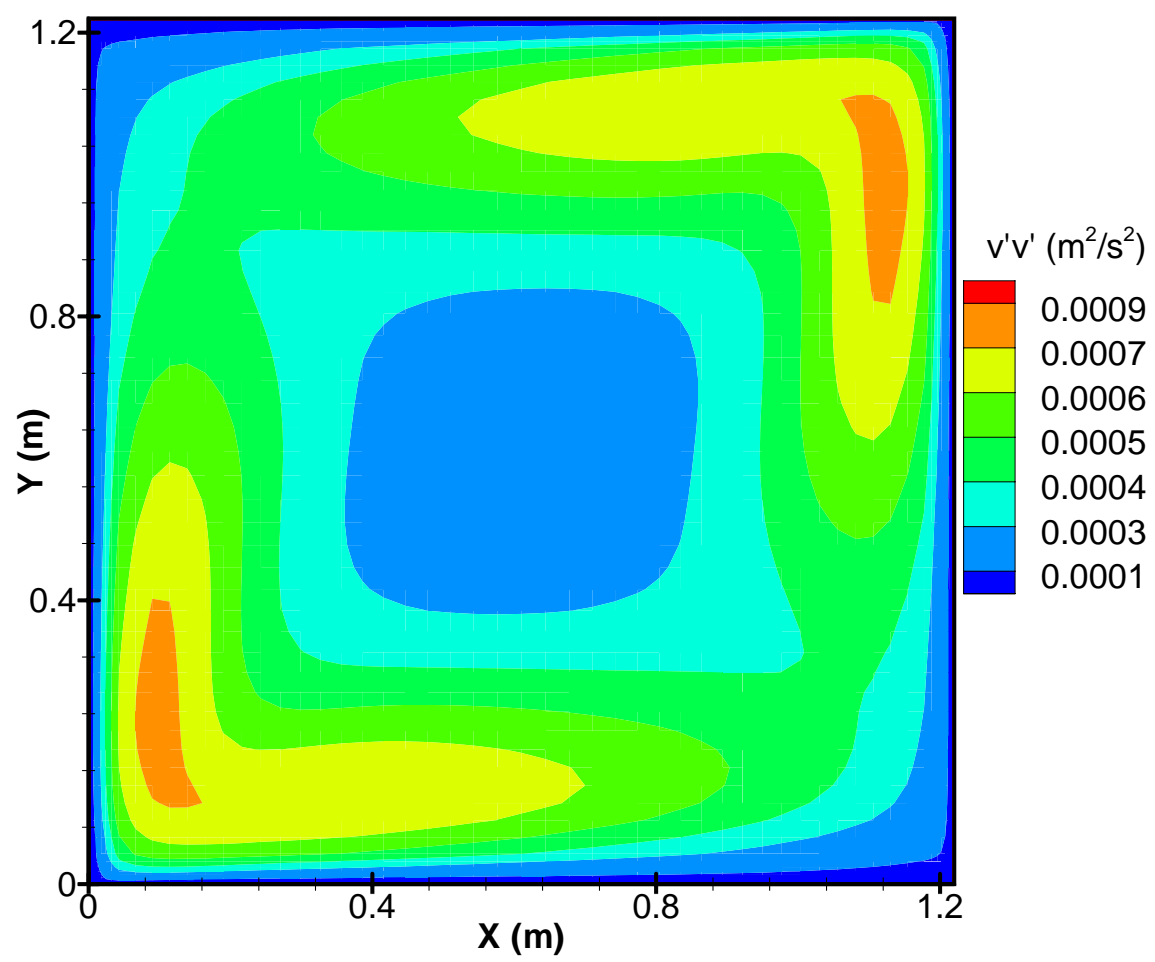

Figure 6. Predicted turbulence characteristics in the cavity: (a) turbulence kinetic energy, (b) wall-normal turbulence fluctuation $\overline{v^{\prime 2}}$ by the v2f-dav model. 
(a)

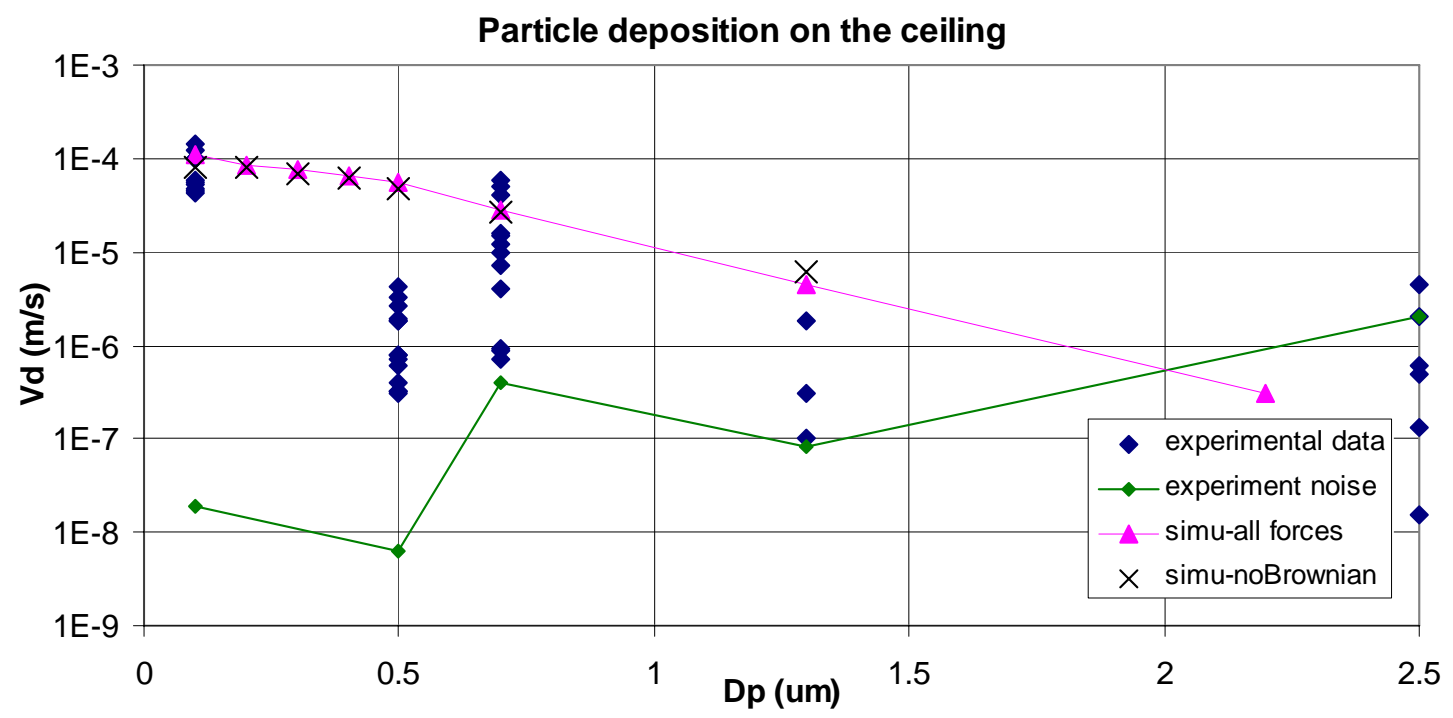

(b)

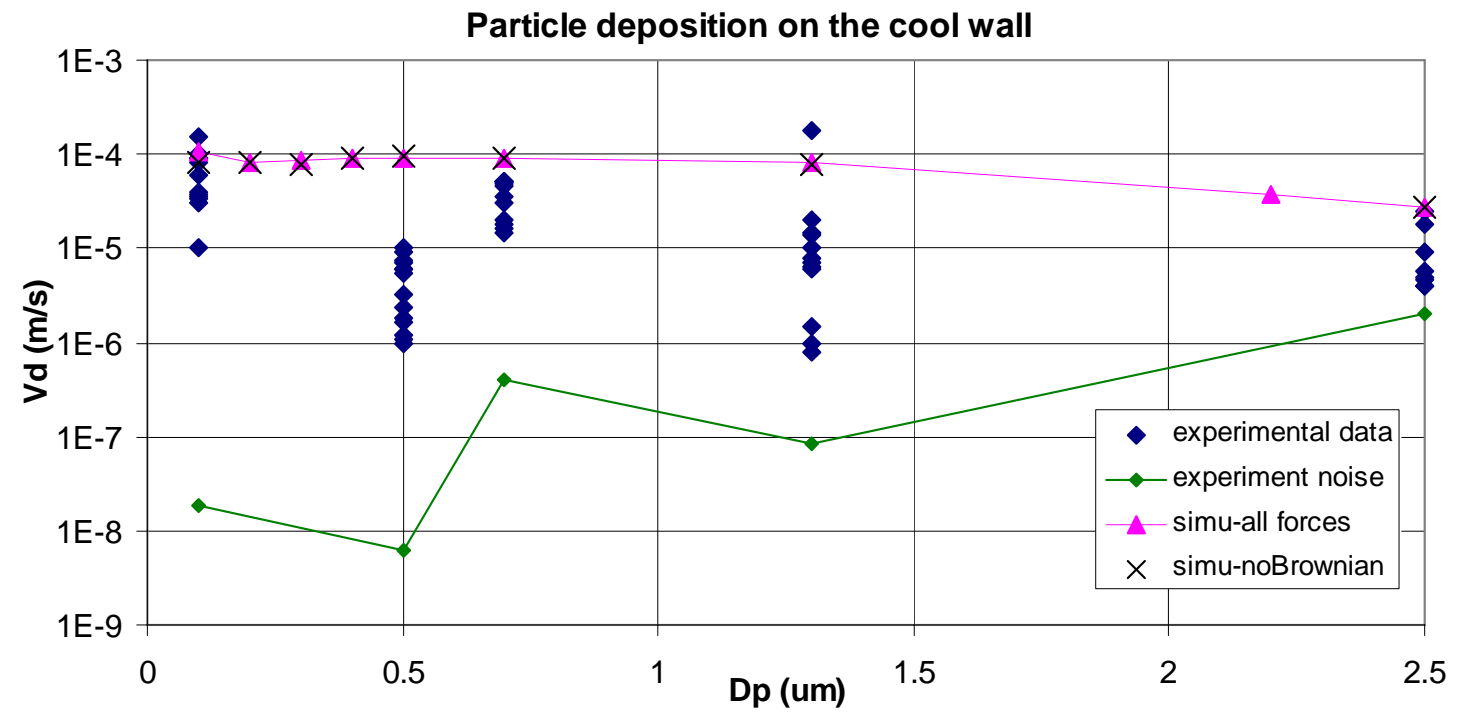

Figure 7. Comparison of predicted and measured particle deposition velocity on different surfaces: (a) on the ceiling, (b) on the cool wall, (c) on the floor, (d) on the warm wall. 
(c)

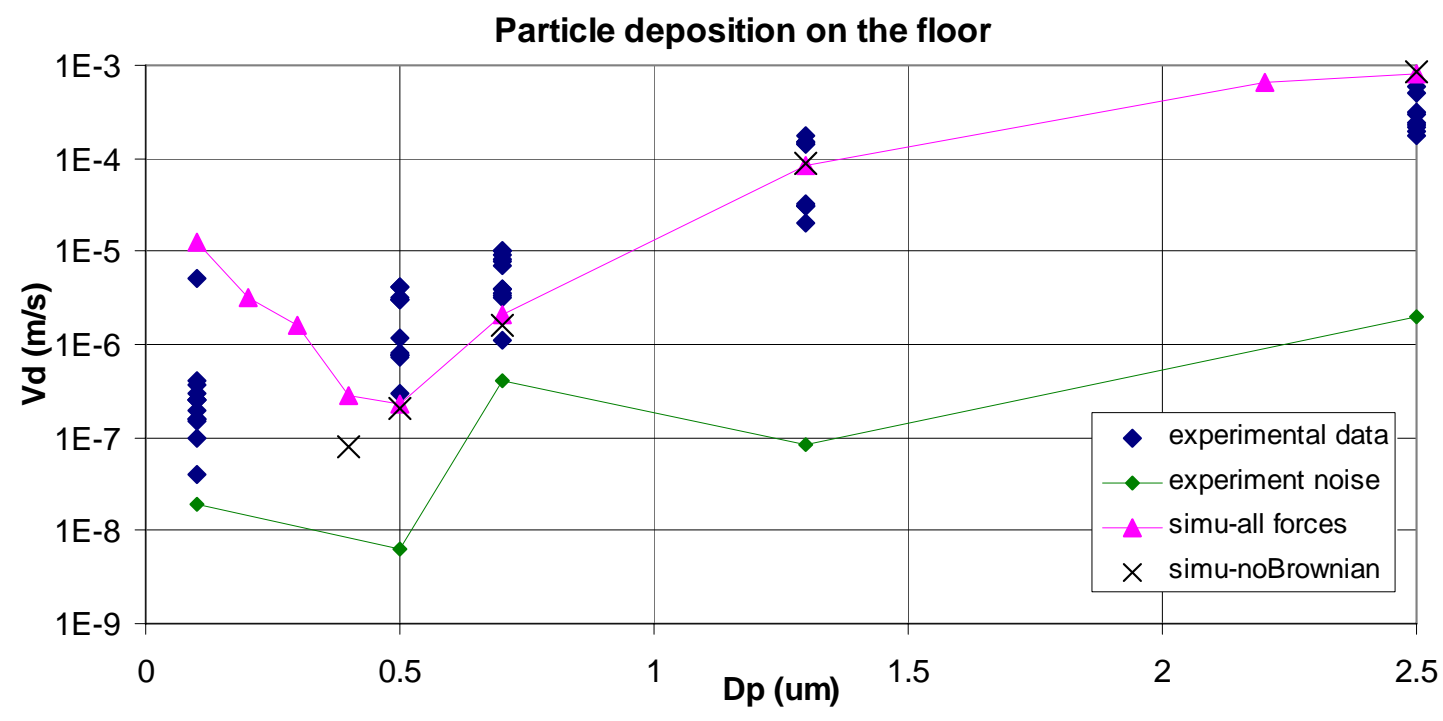

(d)

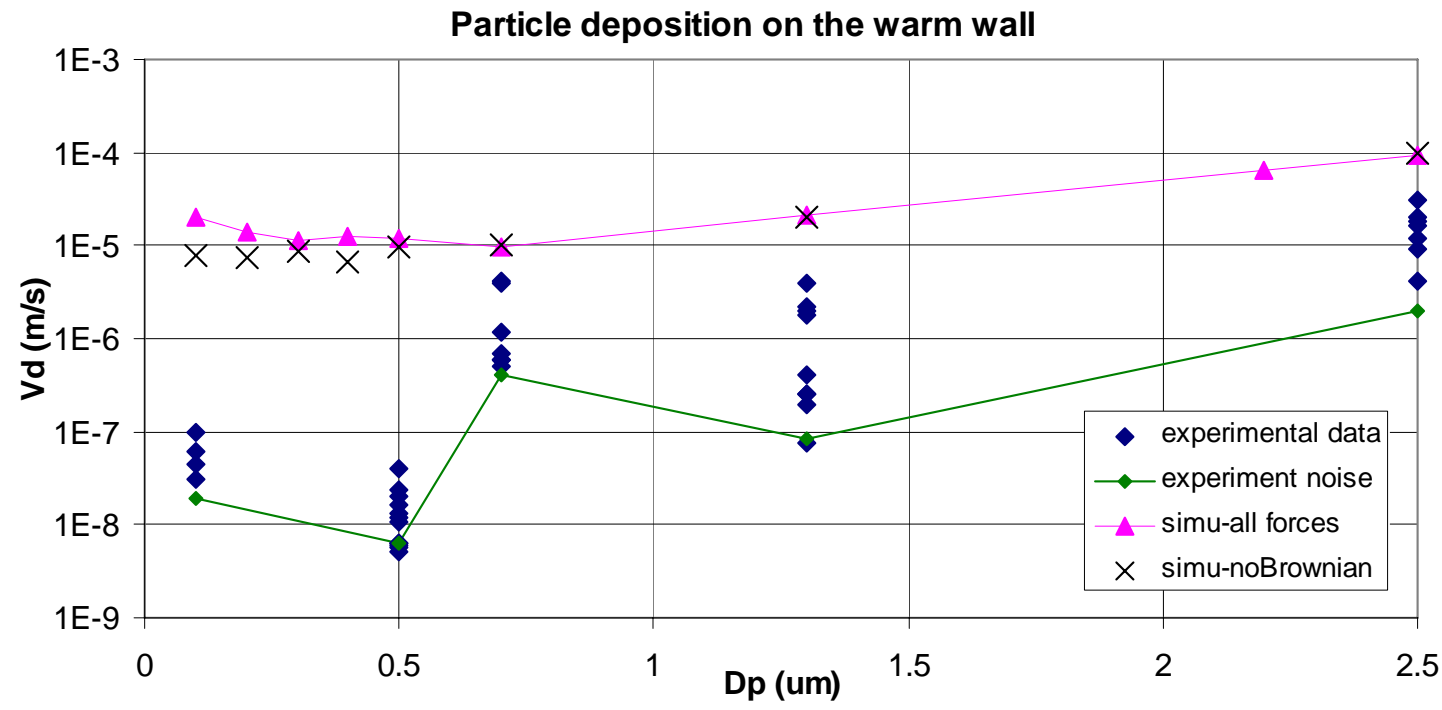

Figure 7. Comparison of predicted and measured particle deposition velocity on different surfaces: (a) on the ceiling, (b) on the cool wall, (c) on the floor, (d) on the warm wall. 
(a)

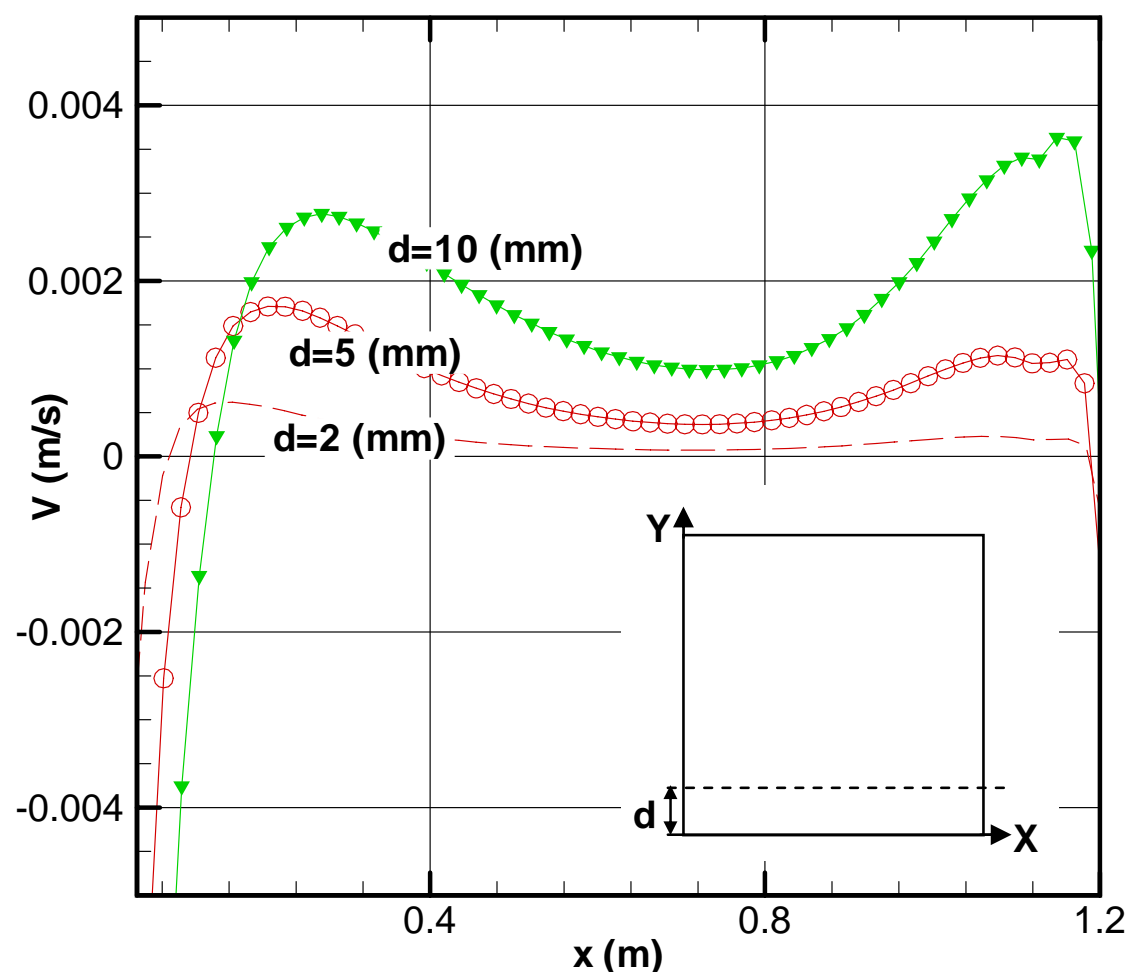

(b)

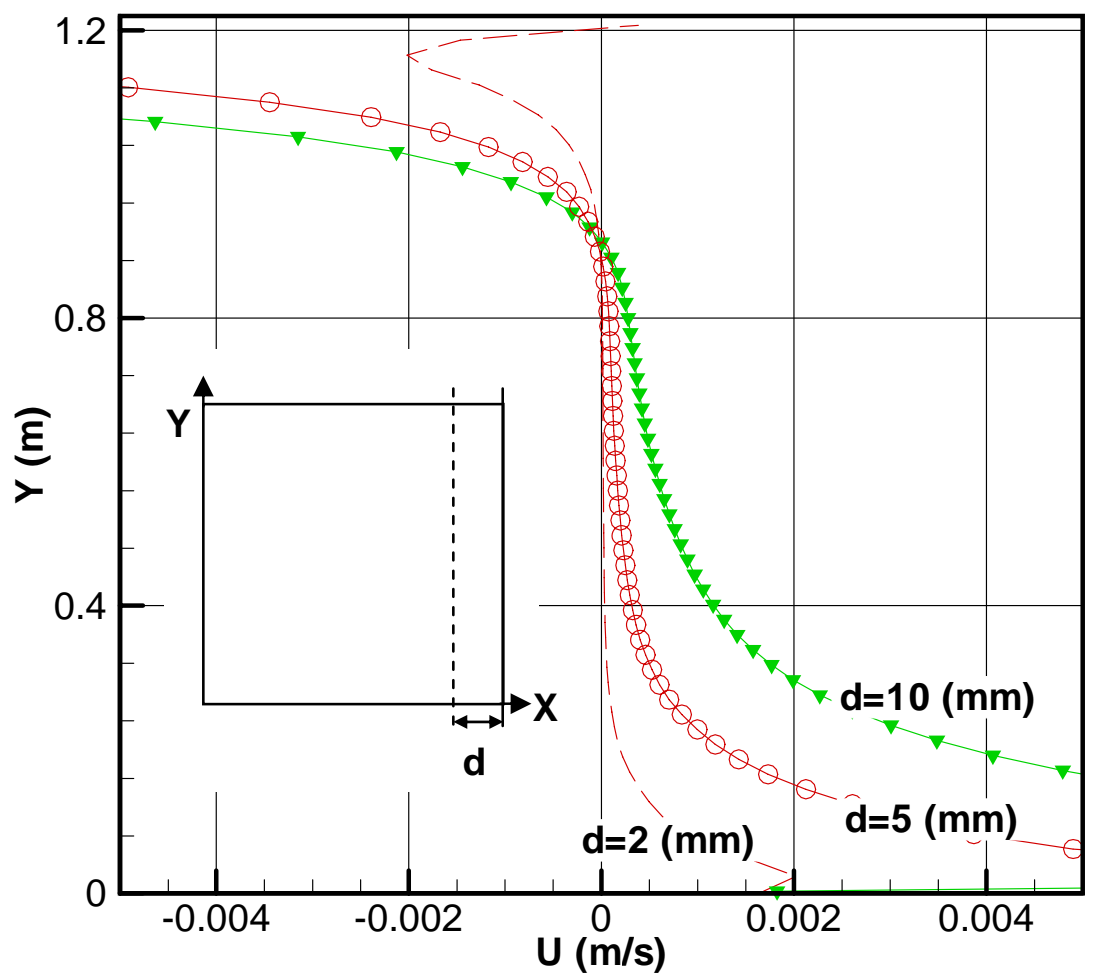

Figure 8. Predicted wall normal velocity near surfaces at different distances: (a) near the warm floor, (b) near the warm wall. 